\title{
Empiric Antibiotic Regimens for Neonatal Sepsis in Australian and New Zealand Neonatal Intensive Care Units.
}

\section{Original Article}

\section{Jeremy Carr', David Burgner1,2,3,4, Rohan Hardikar ${ }^{5}$, Jim Buttery 1,2,3,4,6}

1. Dept. of Infection and Immunity, Monash Children's, Melbourne Australia

2. Dept. of Paediatrics, Monash University, Australia

3. Murdoch Children's Research Institute, Melbourne Australia

4. Dept. of Paediatrics, University of Melbourne, Australia

5. Southern Clinical School, Monash University, Australia

6. The Ritchie Centre, Hudson Institute of Medical Research, Monash University, Australia

Corresponding Author

Jeremy Carr

Infectious Diseases Unit

Royal Children's Hospital Melbourne

Flemington Road, Parkville, 3052 Vic, Australia

$\mathrm{Ph}+61393455522$

Jeremy.carr@rch.org.au

This is the author manuscript accepted for publication and has undergone full peer review but has not been through the copyediting, typesetting, pagination and proofreading process, which may lead to differences between this version and the Version of Record. Please cite this article as doi: $10.1111 /$ jpc. 13540

This article is protected by copyright. All rights reserved. 


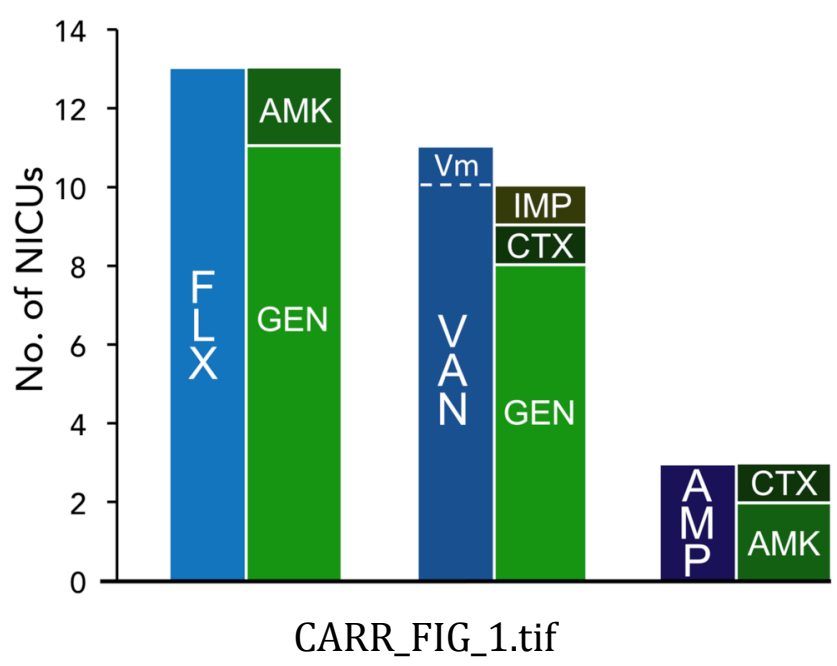

This article is protected by copyright. All rights reserved. 


\section{Empiric Antibiotic Regimens for Neonatal Sepsis in Australian and New Zealand Neonatal Intensive Care Units}

\section{Abstract}

Aim: Neonatal Sepsis remains an important cause of morbidity and mortality, and requires prompt empiric treatment. However only a minority of babies who receive antibiotics for suspected sepsis have an infection. Antimicrobial exposure in infancy has important short and long-term consequences. There is no consensus regarding empirical antimicrobial regimens.

Methods: Survey of empiric antimicrobial regimens in all tertiary neonatal intensive care units in Australia and New Zealand in 2013-14.

Results: All 27 units responded. For Early-onset Sepsis, all units used a combination of gentamicin with either penicillin or ampicillin. For Late-onset Sepsis, the frequency of units using empiric vancomycin (41\%) versus empiric flucloxacillin (48\%) was similar. Gestational age or the presence of a central venous catheter had little influence on using vancomycin instead of flucloxacillin. For Late-onset Sepsis with meningitis there was marked variation in antimicrobial combinations, with 15 different regimens described. 93\% used a cefotaxime-based regimen, either as monotherapy (22\%), or combined with a second (22\%) or third (48\%) agent. For suspected necrotising enterocolitis, $89 \%$ used an aminoglycoside, metronidazole and a penicillin. Historical outbreaks of multi-resistant organisms exerted long-term influence over regimen choice.

Conclusions: There was limited use of broad-spectrum agents such as carbapenems or third-generation cephalosporins. In this region with low MRSA prevalence, empiric vancomycin use was common, selected for activity against coagulase-negative staphylococci (CoNS). Empiric vancomycin is rarely necessary because CoNS are often contaminants and sepsis is rarely fulminant, occurring almost exclusively in extremely-low birth-weight infants. Implementation of appropriate, local antimicrobial policies is crucial to minimise antimicrobial exposure in this vulnerable population and halt the development of antimicrobial resistance. 
What is already known on this topic

- Optimal empiric antimicrobial regimens for common neonatal infections are not well defined

- The majority of infants who commence antibiotics for suspected sepsis do not have an infection.

- Broad-spectrum antimicrobial use facilitates antibiotic resistance and additionally may have long-term health consequences through modulation of the early-life microbiome

\section{What this paper adds}

- Highlights the variation in antimicrobial regimens across all Australian and New Zealand tertiary Neonatal Intensive Care Units

- Demonstrates that the use of broad-spectrum antibiotics is limited, although the inclusion of empiric vancomycin is common but contentious.

- Reinforces the need for consistent antimicrobial policies to limit the development of antimicrobial resistance and safely minimise broad-spectrum antimicrobial exposure in the critical infant period.



This article is protected by copyright. All rights reserved. 


\section{Introduction}

Sepsis remains an important cause of neonatal morbidity and mortality, and necessitates prompt recognition and treatment. Clinical signs are non-specific and laboratory investigations lack negative predictive value to confidently refute the presence of infection. Consequently, most infants who commence empiric antimicrobial therapy do not have an infection.[1, 2] Antimicrobial use promotes colonisation with resistant bacteria, predisposes to necrotising enterocolitis (NEC), invasive candidiasis, and may independently contribute to risk of death.[3-5] Exposure to antimicrobials in infancy may have broad and pervasive influence on life-long risk of both communicable and non-communicable disease through modulation of the early-life microbiome. [6, 7] Appropriate empiric antimicrobial regimens combined with antimicrobial stewardship programs (ASP) may minimise adverse consequences. Implementation is challenging in light of lack of consensus on clinical sepsis definitions, the differing empiric regimens used and paucity of evidence for commonly included broad-spectrum antibiotics such as vancomycin or $3^{\text {rd }}$ generation cephalosporins.[8-15]

The Australian and New Zealand Neonatal Network (ANZNN) is a collaborative body of all neonatal intensive care units in Australia and New Zealand that monitors outcomes from high-risk neonates. Crude rates of infection are included, but not include organism and susceptibility data or antimicrobial use. We surveyed all tertiary Neonatal Intensive Care Units (NICUs) of the ANZNN to characterise empiric antimicrobial regimens for common neonatal infections.

\section{Methods}

An on-line REDCAP-based[16] survey of unit-wide antimicrobial policies was distributed to all tertiary NICUs in Australian and New Zealand in 2013-14. Permission to conduct the survey was obtained from the ANZNN, and a consultant neonatologist completed the survey at each site (head of unit or designated consultant responsible for infection). The questionnaire surveyed empiric antibiotic regimens for EOS, LOS, and NEC, assuming that microbiology results for each scenario were not yet available (online supplement). LOS regimens were stratified as 
'meningitis' or 'not meningitis' based on abnormal cerebrospinal fluid (CSF) microscopy and biochemistry, although abnormal CSF parameters were intentionally not defined, given lack of consensus. The survey included modifications to empiric regimens, based on factors such as birth weight, gestational age or the presence of a central line. Diagnostic methods, treatment duration, local epidemiology and antimicrobial resistance patterns were outside the scope of this questionnaire.

\section{Results}

All 27 tertiary NICUs in Australia (21) and New Zealand (6) completed the survey. The cut-off between EOS and LOS was defined as clinical infection developing $\geq 48$ hours after birth in $63 \%$ of units, as $\geq 72$ hours in $26 \%$ and $\geq 7$ days of life in $11 \%$.

Early-onset sepsis (EOS): All units used gentamicin in combination with either benzylpenicillin (67\%) or ampicillin (30\%), with one unit allowing consultant discretion between choosing ampicillin or penicillin. No unit used cephalosporins or carbapenems in their first-line empiric EOS regimen.

Late-onset sepsis (LOS) without meningitis: There were eight different empiric antibiotic regimens across the 27 NICUs (Figure 1). The frequency of empiric use of flucloxacillin (48\%) was similar to vancomycin (41\%). Gram-negative coverage was most often an aminoglycoside (85\%, either gentamicin (70\%), or amikacin (15\%)) followed by cefotaxime (7\%) or imipenem (4\%). One unit specified the use of vancomycin monotherapy if central line infection was suspected, and added either cefotaxime or gentamicin if the baby was clinically 'unwell', or alternatively commenced piperacillin-tazobactam.

LOS with presumed meningitis: There were 15 different regimens amongst the 27 units. The complexity of regimen choice is depicted schematically in Figure 2. Ninetythree per cent of NICUs used cefotaxime-containing regimens. Of these, six (22\%) used cefotaxime monotherapy, while 19 (70\%) combined cefotaxime with a second (22\%) and third (48\%) antimicrobial. The difference between regimens chosen for LOS with and without meningitis for each unit varied. Nine NICUs added cefotaxime 
to their standard LOS regimens, three substituted an aminoglycoside for cefotaxime, and four units modified empiric LOS regimens to include either ampicillin or penicillin. Two units used non-cefotaxime based regimens: one unit used ceftazidime due to a period of higher incidence of pseudomonas sepsis three years before the survey; the other unit used imipenem and vancomycin for LOS with or without meningitis. This regimen was initiated in the context of a resolved outbreak of ESBL producing Klebsiella pneumoniae eighteen years prior to the survey but had remained the current first-line recommendation.

Modifications to empiric LOS regimens: Of the 13 NICUs who used empiric flucloxacillin for LOS, only three would switch to vancomycin in the presence of a central venous catheter. For LOS, one unit used flucloxacillin for infants with corrected age above 29 weeks, and vancomycin in infants under 29 weeks corrected age. No NICU modified empiric antibiotics according to birth weight.

Necrotising enterocolitis (NEC): Eight-nine per cent of units prescribed an aminoglycoside and metronidazole, combined with ampicillin (44\%), penicillin (11\%), flucloxacillin (15\%) or vancomycin (19\%). Two units (7\%) used piperacillintazobactam monotherapy and one unit used gentamicin combined with amoxycillinclavulanate. No unit used clindamycin.

\section{Discussion}

This survey of all NICUs in Australian and New Zealand highlights variance in empiric antimicrobial regimens for common neonatal infections. This is most pronounced for LOS with meningitis, with fifteen different antibiotic regimens across 27 units. In the absence of robust evidence from randomised controlled trials, previous surveys have also highlighted the variation in antimicrobial use, particularly the inclusion of vancomycin or broad-spectrum cephalosporins.[8, 10, 14, 15, 17]

The temporal cut-off between EOS and LOS (to denote vertical versus nosocomial infection) is indistinct and published definitions vary between 48,72 hours or 7 
days.[12, 18-20] Our regional survey reflected this lack of consensus, despite the ANZNN distinguishing between EOS and LOS at 48 hours.[21]

All EOS regimens provided adequate coverage for the major pathogens, including Listeria, and no NICU used vancomycin or a cephalosporin. The causative organisms of LOS in Australia and New Zealand tertiary NICUs are similar to those described from other developed settings, although antimicrobial susceptibilities vary.[1, 22] Coagulase-negative staphylococci (CoNS) account for approximately half of all culture-positive episodes.[1, 22] For LOS, most units used an aminoglycoside in combination with either flucloxacillin or vancomycin in equal proportion. Two units (7\%) did not use an empiric regimen with activity against methicillin-sensitive Staphylococcus aureus (MSSA), while one other unit relied upon cefotaxime to cover MSSA. A 2003 survey of several Australian NICUs also showed an equal proportion of units using either vancomycin or flucloxacillin, whilst in a recent European survey only $15 \%$ of units included vancomycin in empiric LOS regimens.[17, 23] Our survey was conducted in region with a low prevalence of methicillin-resistant Staphylococcus aureus, [24] thus the rationale for empiric vancomycin is primarily for CoNS, as over $90 \%$ of isolates are resistant to flucloxacillin.[22, 25] Although CoNS accounts for over $50 \%$ of LOS isolates in some series, their true contribution to sepsis is not clearly defined because differentiation between CoNS as a contaminant or a pathogen is problematic and definitions vary between studies. $[1,22,26]$ The rate of neonatal Staphylococcal infection is also significantly influenced by infection control and prevention practices.[27] CoNS are low virulence organisms typically causing indolent disease, with fulminant sepsis occurring in less than $1 \%$ of cases[28] and deaths attributed to CoNS are almost exclusively in extremely low birth weight infants.[26] CoNS-related mortality is difficult to estimate but is lower than previous studies suggested, with combined direct and indirect attributable mortality ranging from $0.33 \%-5 \% .[23,26,28,29]$

No studies have demonstrated a benefit of empiric vancomycin for CoNS sepsis, although existing studies are mostly retrospective and underpowered to detect a low attributable mortality of CoNS sepsis.[23, 30-34] Adverse effects associated with 
vancomycin include nephrotoxicity, the selection of vancomycin-resistance in CoNS and Enterococci (VRE), resistant Gram-negative colonisation and, Staphylococcus aureus colonisation and infection.[35-39] Neonatal vancomycin dosing is problematic and there is often delay in obtaining therapeutic levels, which is thought to correlate with both treatment failure and the development of resistance. In considering the uncertain benefit of vancomycin with potential adverse consequences, a successful and safe strategy to limit vancomycin use in most cases is delaying the initiation of vancomycin until identification of a clinically-significant CoNS in blood culture.[31]

Aminoglycosides are the mainstay for empiric Gram-negative coverage in Australia and New Zealand. Only seven per cent of NICUs used an empiric $3^{\text {rd }}$ generation cephalosporin for LOS without meningitis. The use of a cephalosporin confers no benefit in antimicrobial spectrum over a beta-lactam/aminoglycoside combination.[40] Several studies have demonstrated an association between cephalosporin use and increased rates of antimicrobial resistance within units, and some indicate increased morbidity or mortality.[5, 41]

Variation between units was particularly evident when meningitis was suspected, and the complexity of antimicrobial combinations, depicted in Figure 2, cannot be explained by antimicrobial spectrum or likely susceptibility patterns. One in five units did not cover Listeria infection. The use of dual Gram-negative agents, particularly the combination of an aminoglycoside and a third-generation cephalosporin was common. There is no literature supporting the use of dual Gram-negative antimicrobials in meningitis. The use of 'synergistic' gentamicin in meningitis caused by Gram-positive organisms is based solely on in vitro models and animal studies, with no difference in clinical outcomes in infants.

Antibiotic regimens for presumed NEC differed predominantly in choice of Grampositive cover. The inclusion of flucloxacillin by some units relates to the low discrimination between the signs of early NEC and septic ileus, where Staphylococcus aureus is a potential (and fulminant) pathogen.[24, 42] The use of vancomycin may reflect the known association between CoNS and NEC. Similar to CoNS sepsis, NEC 
associated with CoNS bacteraemia has a low mortality.[43] The long-term effects of the beta-lactam/beta-lactamase inhibitor combination piperacillin-tazobactam on the development of resistance are not well studied. A recent Cochrane review concluded there was insufficient evidence to recommend a specific antibiotic regimen for NEC.[44]

The strengths of this study include that this is the first regional survey of neonatal antibiotic practice in tertiary NICUs and had a complete response rate. We acknowledge some inevitable shortcomings, including that surveying a single neonatologist for each unit may not adequately capture within-unit differences in practice, despite the survey stipulating unit-wide policies or practice. Our survey does not assess actual antibiotic use[45], adherence to empiric protocol, or local antimicrobial stewardship resources, which are important factors in reducing antibiotic exposure. Neonatal units constitute a significant proportion of paediatric inpatient antimicrobial use and the majority of antibiotic use for suspected sepsis will follow institutional protocol. Thus defining the optimal empiric regimen is an integral component of neonatal antimicrobial stewardship. Further research should clarify the role of vancomycin in LOS, including the efficacy of delayed or stratified use, although an adequately powered study would require a large study population. The persistent use of ceftazidime and imipenem following outbreaks of resistant organisms many years earlier revealed in this survey highlights the need for timely and regular review of outbreak control strategies to return to a more narrowspectrum regimen as soon as safely possible.

The use of broad-spectrum antibiotics in empiric regimens in Australia and New Zealand is relatively limited, with the exception of vancomycin, which has a limited empiric role in settings with low MRSA prevalence. Currently, there is a low prevalence of multi-resistant organisms in Australian and New Zealand neonatal units. In Asia, there is widespread resistance in Gram-negative organisms to first line antibiotics, ampicillin and gentamicin, as well as cephalosporins.[46, 47] Similar patterns of resistance are seen in many other regions.[48] There is a time-critical opportunity to preserve the low rate of antimicrobial resistance in Australia and New 
Zealand. Development and broad implementation of evidence-based empiric antimicrobial regimens informing both choice and duration of therapies, together with recent successes in antimicrobial stewardship programs and the reduction in line related blood-stream infections using multi-component " bundled" interventions, should better protect our most vulnerable newborns.

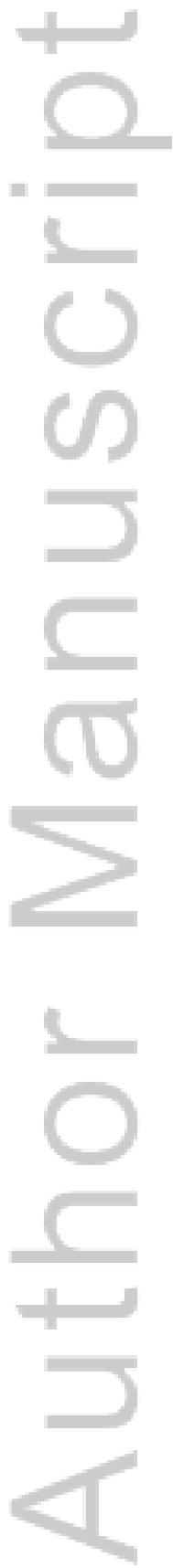

This article is protected by copyright. All rights reserved. 


\section{Acknowledgements}

The authors acknowledge the contribution of Dr Alice Stewart at Monash Newborn, and thank A/Prof Ross Haslam, Chair of the Australian and New Zealand Neonatal Network. We also thank all the survey participants. Australia: Zsuzsoka Kecskes, The Canberra Hospital, ACT; Nick Evans, Royal Prince Alfred Hospital, NSW; Amit Trivedi, The Children's Hospital at Westmead, NSW; Jacqueline Stack, Liverpool Health Service, NSW. Paul Craven, John Hunter Children's Hospital, NSW; Mary Paradisis, Royal North Shore Hospital, NSW; Ulrike Brandenburg, Nepean Hospital, NSW; Meredith Ward, Royal Hospital for Women, NSW; Ajit Aiyappan, Royal Darwin Hospital, NT; David Knight, Mater Mothers' Hospital, QLD; David Cartwright, Royal Brisbane \& Women's, QLD; Guan Koh, Townsville Hospital, QLD; Sanjay Sinhal \& Peter Marshall, Flinders Medical Centre, SA; Vinish Bhatia, Women's and Children's Hospital Adelaide, SA; Peter Dargaville, Royal Hobart Hospital, TAS; Elizabeth Carse, Monash Medical Centre, VIC; Carl Kuschel, The Royal Women's Hospital, VIC; Andrew Watkins, Mercy Hospital for Women, VIC; Neoil Patel, Royal Children's Hospital Melbourne, Vic; Shripada Rao, Princess Margaret Hospital for Children, WA; Tobias Strunk, King Edward Memorial Hospital, WA. New Zealand: David Bourchier, Waikato Hospital; Lindsay Mildenhall, Middlemore Hospital; Nicola Austin, Christchurch Women's Hospital; Roland Broadbent, Dunedin Hospital; Malcolm Battin, National Women's Health, Auckland; Max Berry, Wellington Women's Hospital.

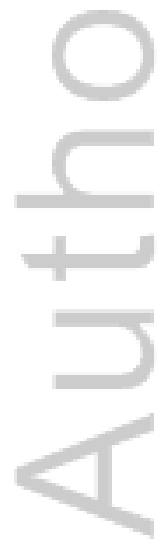




\section{References}

[1] Lean WL, Kamlin CO, Garland SM, Jacobs SE. Stable rates of neonatal sepsis in a tertiary neonatal unit. J Paediatr Child Health. 2015; 51: 294-299.

[2] Escobar GJ, Li DK, Armstrong MA, et al. Neonatal sepsis workups in infants $\geq$ 2000 grams at birth: A population-based study. Pediatrics. 2000; 106: 256-263.

[3] Cotten CM, Taylor S, Stoll B, et al. Prolonged duration of initial empirical antibiotic treatment is associated with increased rates of necrotizing enterocolitis and death for extremely low birth weight infants. Pediatrics. 2009; 123: 58-66. [4] Kuppala VS, Meinzen-Derr J, Morrow AL, Schibler KR. Prolonged initial empirical antibiotic treatment is associated with adverse outcomes in premature infants. J Pediatr. 2011; 159: 720-725.

[5] de Man P, Verhoeven BA, Verbrugh HA, Vos MC, van den Anker JN. An antibiotic policy to prevent emergence of resistant bacilli. Lancet. 2000; 355: $973-$ 978.

[6] Saari A, Virta LJ, Sankilampi U, Dunkel L, Saxen H. Antibiotic exposure in infancy and risk of being overweight in the first 24 months of life. Pediatrics. 2015; 135: 617-626.

[7] Groer MW, Luciano AA, Dishaw L, Ashmeade TL, Miller E, Gilbert JA. Development of the preterm infant gut microbiome: a research priority. Microbiome. 2014; 2: 38.

[8] Gordon A, Jeffery HE. Antibiotic regimens for suspected late onset sepsis in newborn infants. Cochrane Database Syst Rev. 2005: CD004501.

[9] Wynn JL, Wong HR, Shanley TP, Bizzarro MJ, Saiman L, Polin RA. Time for a neonatal-specific consensus definition for sepsis. Pediatr Crit Care Med. 2014; 15: 523-528.

[10] van Herk W, el Helou S, Janota J, et al. Variation in Current Management of Term and Late-preterm Neonates at Risk for Early-onset Sepsis: An International Survey and Review of Guidelines. Pediatr Infect Dis J. 2016; 35: 494-500.

[11] Cantey JB, Wozniak PS, Sanchez PJ. Prospective surveillance of antibiotic use in the neonatal intensive care unit: results from the SCOUT study. Pediatr Infect Dis J. 2015; 34: 267-272.

[12] Liem TB, Krediet TG, Fleer A, Egberts TC, Rademaker CM. Variation in antibiotic use in neonatal intensive care units in the Netherlands. $J$ Antimicrob Chemother. 2010; 65: 1270-1275.

[13] Grohskopf LA, Huskins WC, Sinkowitz-Cochran RL, et al. Use of antimicrobial agents in United States neonatal and pediatric intensive care patients. Pediatr Infect Dis J. 2005; 24: 766-773.

[14] Fernando AM, Heath PT, Menson EN. Antimicrobial policies in the neonatal units of the United Kingdom and Republic of Ireland. J Antimicrob Chemother. 2008; 61: 743-745.

[15] Rubin LG, Sanchez PJ, Siegel J, et al. Evaluation and treatment of neonates with suspected late-onset sepsis: a survey of neonatologists' practices. Pediatrics. 2002; 110: e42.

[16] Harris PA, Taylor R, Thielke R, Payne J, Gonzalez N, Conde JG. Research electronic data capture (REDCap)--a metadata-driven methodology and workflow process for providing translational research informatics support. $J$ Biomed Inform. 2009; 42: 377-381. 
[17] Spyridis N, Syridou G, Goossens H, et al. Variation in paediatric hospital antibiotic guidelines in Europe. Arch Dis Child. 2016; 101: 72-76.

[18] van der Zwet WC, Kaiser AM, van Elburg RM, et al. Nosocomial infections in a Dutch neonatal intensive care unit: surveillance study with definitions for infection specifically adapted for neonates. J Hosp Infect. 2005; 61: 300-311.

[19] Li Z, Xiao Z, Li Z, Zhong Q, Zhang Y, Xu F. 116 cases of neonatal early-onset or late-onset sepsis: A single center retrospective analysis on pathogenic bacteria species distribution and antimicrobial susceptibility. Int J Clin Exp Med. 2013; 6: 693699.

[20] Jiang JH, Chiu NC, Huang FY, et al. Neonatal sepsis in the neonatal intensive care unit: characteristics of early versus late onset. J Microbiol Immunol Infect. 2004; 37: 301-306.

[21] Australian and New Zealand Neonatal Network Data Dictionary 2015. 1-180. http://www.anznn.net accessed 28/5/2016.

[22] Isaacs D, Barfield C, Clothier T, et al. Late-onset infections of infants in neonatal units. J Paediatr Child Health. 1996; 32: 158-161.

[23] Isaacs D. A ten year, multicentre study of coagulase negative staphylococcal infections in Australasian neonatal units. Arch Dis Child Fetal Neonatal Ed. 2003; 88: F89-93.

[24] Isaacs D. Staphylococcus aureus infections in Australasian neonatal nurseries. Arch Dis Child Fetal Neonatal Ed. 2004; 89: F331-335.

[25] Qu Y, Daley AJ, Istivan TS, Garland SM, Deighton MA. Antibiotic susceptibility of coagulase-negative staphylococci isolated from very low birth weight babies:

comprehensive comparisons of bacteria at different stages of biofilm formation. Ann Clin Microbiol Antimicrob. 2010; 9: 16.

[26] Healy CM, Baker CJ, Palazzi DL, Campbell JR, Edwards MS. Distinguishing true coagulase-negative Staphylococcus infections from contaminants in the neonatal intensive care unit. J Perinatol. 2013; 33: 52-58.

[27] Bizzarro MJ, Shabanova V, Baltimore RS, Dembry LM, Ehrenkranz RA, Gallagher PG. Neonatal sepsis 2004-2013: the rise and fall of coagulase-negative staphylococci. J Pediatr. 2015; 166: 1193-1199.

[28] Karlowicz MG, Buescher ES, Surka AE. Fulminant late-onset sepsis in a neonatal intensive care unit, 1988-1997, and the impact of avoiding empiric vancomycin therapy. Pediatrics. 2000; 106: 1387-1390.

[29] Healy CM, Palazzi DL, Edwards MS, Campbell JR, Baker CJ. Features of invasive staphylococcal disease in neonates. Pediatrics. 2004; 114: 953-961. [30] Krediet TG, Jones ME, Gerards LJ, Fleer A. Clinical outcome of cephalothin versus vancomycin therapy in the treatment of coagulase-negative staphylococcal septicemia in neonates: relation to methicillin resistance and mec A gene carriage of blood isolates. Pediatrics. 1999; 103: E29.

[31] Chiu CH, Michelow IC, Cronin J, Ringer SA, Ferris TG, Puopolo KM.

Effectiveness of a guideline to reduce vancomycin use in the neonatal intensive care unit. Pediatr Infect Dis J. 2011; 30: 273-278.

[32] Lawrence SL, Roth V, Slinger R, Toye B, Gaboury I, Lemyre B. Cloxacillin versus vancomycin for presumed late-onset sepsis in the Neonatal Intensive Care Unit and the impact upon outcome of coagulase negative staphylococcal bacteremia: a retrospective cohort study. BMC Pediatr. 2005; 5: 49. 
[33] Matrai-Kovalskis Y, Greenberg D, Shinwell ES, Fraser D, Dagan R. Positive blood cultures for coagulase-negative staphylococci in neonates: does highly selective vancomycin usage affect outcome? Infection. 1998; 26: 85-92.

[34] Ericson JE, Thaden J, Cross HR, et al. No survival benefit with empirical vancomycin therapy for coagulase-negative staphylococcal bloodstream infections in infants. Pediatr Infect Dis J. 2015; 34: 371-375.

[35] Rasigade JP, Raulin O, Picaud JC, et al. Methicillin-resistant Staphylococcus capitis with reduced vancomycin susceptibility causes late-onset sepsis in intensive care neonates. PLoS One. 2012; 7: e31548.

[36] Ofek-Shlomai N, Benenson S, Ergaz Z, Peleg O, Braunstein R, Bar-Oz B. Gastrointestinal colonization with ESBL-producing Klebsiella in preterm babies--is vancomycin to blame? Eur J Clin Microbiol Infect Dis. 2012; 31: 567-570.

[37] Van Houten MA, Uiterwaal CS, Heesen GJ, Arends JP, Kimpen JL. Does the empiric use of vancomycin in pediatrics increase the risk for Gram-negative bacteremia? Pediatr Infect Dis J. 2001; 20: 171-177.

[38] Rubin LG, Tucci V, Cercenado E, Eliopoulos G, Isenberg HD. Vancomycinresistant Enterococcus faecium in hospitalized children. Infect Control Hosp Epidemiol. 1992; 13: 700-705.

[39] Recommendations for preventing the spread of vancomycin resistance. Recommendations of the Hospital Infection Control Practices Advisory Committee (HICPAC). MMWR Recomm Rep. 1995; 44: 1-13.

[40] Russell AB, Sharland M, Heath PT. Improving antibiotic prescribing in neonatal units: time to act. Arch Dis Child Fetal Neonatal Ed. 2012; 97: F141-146. [41] Clark RH, Bloom BT, Spitzer AR, Gerstmann DR. Empiric use of ampicillin and cefotaxime, compared with ampicillin and gentamicin, for neonates at risk for sepsis is associated with an increased risk of neonatal death. Pediatrics. 2006; 117: 67-74. [42] Chan KL, Saing H, Yung RW, Yeung YP, Tsoi NS. A study of pre-antibiotic bacteriology in 125 patients with necrotizing enterocolitis. Acta Paediatr Suppl. 1994; 396: 45-48.

[43] Saenz de Pipaon Marcos M, Rodriguez Delgado J, Martinez Biarge M, et al. Low mortality in necrotizing enterocolitis associated with coagulase-negative Staphylococcus infection. Pediatr Surg Int. 2008; 24: 831-835.

[44] Shah D, Sinn JK. Antibiotic regimens for the empirical treatment of newborn infants with necrotising enterocolitis. Cochrane Database Syst Rev. 2012: CD007448. [45] Osowicki J, Gwee A, Noronha J, et al. Australia-wide Point Prevalence Survey of Antimicrobial Prescribing in Neonatal Units: How Much and How Good? Pediatr Infect Dis J. 2015; 34: e185-190.

[46] Zaidi AK, Huskins WC, Thaver D, Bhutta ZA, Abbas Z, Goldmann DA. Hospitalacquired neonatal infections in developing countries. Lancet. 2005; 365: 1175-1188. [47] Saleem AF, Qamar FN, Shahzad H, Qadir M, Zaidi AK. Trends in antibiotic susceptibility and incidence of late-onset Klebsiella pneumoniae neonatal sepsis over a six-year period in a neonatal intensive care unit in Karachi, Pakistan. Int J Infect Dis. 2013; 17: e961-965.

[48] Downie L, Armiento R, Subhi R, Kelly J, Clifford V, Duke T. Communityacquired neonatal and infant sepsis in developing countries: efficacy of WHO's currently recommended antibiotics--systematic review and meta-analysis. Arch Dis Child. 2013; 98: 146-154. 


\section{Figure Titles \& Legends}

Figure 1. Empiric Late-onset Sepsis regimens (non-meningitis) grouped by primary Gram-positive antibiotic.

Legend: FLX flucloxacillin; VAN vancomycin; AMP ampicillin; GEN gentamicin; AMK amikacin; CTX cefotaxime; IMP imipenem; Vm vancomycin monotherapy \pm gentamicin or cefotaxime.

Figure 2. Late-onset Sepsis with suspected meningitis: Schematic depiction of variance in cefotaxime-based empiric regimens Legend: V vancomycin; F flucloxacillin; AG aminoglycoside; P/A penicillin or ampicillin 


\section{University Library}

\section{- M M N E R VA A gateway to Melbourne's research publications}

Minerva Access is the Institutional Repository of The University of Melbourne

Author/s:

Carr, JP;Burgner, DP;Hardikar, RS;Buttery, JP

Title:

Empiric antibiotic regimens for neonatal sepsis in Australian and New Zealand neonatal intensive care units

Date:

2017-07-01

Citation:

Carr, J. P., Burgner, D. P., Hardikar, R. S. \& Buttery, J. P. (2017). Empiric antibiotic regimens for neonatal sepsis in Australian and New Zealand neonatal intensive care units. JOURNAL OF PAEDIATRICS AND CHILD HEALTH, 53 (7), pp.680-684. https://doi.org/10.1111/ jpc. 13540.

Persistent Link:

http://hdl.handle.net/11343/292777 